\title{
Ferret animal model of severe fever with thrombocytopenia syndrome phlebovirus for human lethal infection and pathogenesis
}

\author{
Su-Jin Park ${ }^{1,2,5}$, Young-II Kim ${ }^{1,2,5}$, Angela Park ${ }^{3}$, Hyeok-II Kwon ${ }^{1,2}$, Eun-Ha Kim ${ }^{1,2}$, Young-JaeSi, ${ }^{1,2}$ \\ Min-Suk Song ${ }^{1,2}$, Chul-Ho Lee ${ }^{4}{ }^{4}$, Kyle Jung ${ }^{3}$, Woo-Jin Shin ${ }^{3}{ }^{3}$ Jianxiong Zeng ${ }^{3}$, Younho Choi ${ }^{3}$, \\ JaeU. Jung $\mathbb{1}^{3 \star}$ and Young Ki Choi ${ }^{1,2 \star}$
}

\begin{abstract}
Severe fever with thrombocytopenia syndrome phlebovirus (SFTSV), listed in the most dangerous pathogens by the World Health Organization, has 12-30\% fatality rates with a characteristic thrombocytopenia syndrome. With a majority of clinically diagnosed SFTSV patients older than $\sim \mathbf{5 0}$ years of age, age is a critical risk factor for SFTSV morbidity and mortality. Here, we report an age-dependent ferret model of SFTSV infection and pathogenesis that fully recapitulates the clinical manifestations of human infections. Whereas young adult ferrets ( $\leq 2$ years of age) did not show any clinical symptoms and mortality, SFTSV-infected aged ferrets ( $\geq 4$ years of age) demonstrated severe thrombocytopenia, reduced white blood cell counts and high fever with $93 \%$ mortality rate. Moreover, a significantly higher viral load was observed in aged ferrets. Transcriptome analysis of SFTSV-infected young ferrets revealed strong interferon-mediated anti-viral signalling, whereas inflammatory immune responses were markedly upregulated and persisted in aged ferrets. Thus, this immunocompetent age-dependent ferret model should be useful for anti-SFTSV therapy and vaccine development.
\end{abstract}

\begin{abstract}
n 2009, an emerging infectious disease that caused severe fever, thrombocytopenia and leukocytopenia with a high fatality rate was first reported in China ${ }^{1}$. The disease was named severe fever with thrombocytopenia syndrome (SFTS) and its causative agent, the SFTS phlebovirus (SFTSV) was identified in China in $2010\left(\right.$ refs $\left.^{1-4}\right)$. Since then, the number of SFTS cases has rapidly increased in China, South Korea and Japan ${ }^{5-7}$. Although the average case fatality rate varies between different regions, the mean mortality rates of SFTS cases have remained relatively high in Japan $(27 \%)$, South Korea $(23.3 \%)$ and China $(5.3 \%)^{7-12}$. Thus, the World Health Organization ranked SFTSV among the nine most dangerous pathogens that will most likely cause wide epidemics in the near future, requiring urgent attention ${ }^{13}$. SFTSV belongs to the genus Phlebovirus of the family Phenuiviridae (the order Bunyavirales) ${ }^{2}$ along with other tick-borne Phleboviruses, including Heartland virus and Lone star virus that have been isolated from humans and ticks in the United States ${ }^{14,15}$.

Although there are growing concerns regarding the increasing SFTSV infection rates, limited information regarding its pathogenesis is currently available. This is owing to the lack of an animal model that displays a similar clinical manifestation pattern and high mortality in response to this virus. Previously, type I interferon (IFN)-deficient mice ${ }^{16}$, newborn mice ${ }^{17}$ and mitomycin-treated mice $^{18}$ have all been used in attempts to understand the pathogenesis of SFTSV infection. However, both IFN-deficient and mitomycin-treated mice are immunocompromised and cannot utilize the normal immune response against virus infection. Furthermore, newborn animal models, including mice and hamsters ${ }^{17}$, are also
\end{abstract}

not ideal for studying human SFTSV pathogenesis as the majority of human patients with severe symptoms and increased mortality were more than 50 years of age $\mathrm{e}^{19}$. In addition, in rhesus macaques, SFTSV does not cause severe symptoms or death but causes fever, thrombocytopenia and leukocytopenia, suggesting that rhesus monkey infection resembles only a mild form of SFTSV infection in humans ${ }^{20}$. Thus, the development of an animal model that mimics severe/fatal human SFTS is crucial for understanding the pathogenesis of SFTSV and host immune response to its infection to facilitate the development of medical countermeasures, including vaccines and therapeutics. In this study, we examined the susceptibilities of four different inbred mouse strains and an outbred ferret model (Mustela putorius furo) to SFTSV infection, with separate studies for young adult and aged animals, to establish an animal model of this disease.

\section{Results}

Experimental SFTSV infection in young adult and aged mice and ferrets. To identify an animal model that mimics the clinical manifestation and profile of SFTSV human infection, we examined various mouse strains (BALB/c, C3H, C57BL/6 and FVB) and ferrets following infection with the SFTSV CB1/2014 strain ${ }^{21}$. Groups of 8-week-old mice (young adult mice, $n=5$ ) and 20-24-monthold ferrets (young adult ferrets, $n=5$ ) were intramuscularly (i.m.) inoculated with 0.1 and $1 \mathrm{ml}$ infection volume of stock virus $\left(10^{7.6}\right.$ $\mathrm{TCID}_{50}$ (50\% tissue culture infective dose) of SFTSV CB1/2014), respectively, which was the maximal cell culture dose of this virus. Survival and body weight were recorded for 16 days after inoculation.

'Department of Microbiology, College of Medicine and Medical Research Institute, Chungbuk National University, Cheongju, Republic of Korea. ${ }^{2}$ Zoonotic Infectious Diseases Research Center, Chungbuk National University, Cheongju, Republic of Korea. ${ }^{3}$ Department of Molecular Microbiology and Immunology, Keck School of Medicine, University of Southern California, Los Angeles, CA, USA. ${ }^{4}$ Laboratory Animal Center, Korea Research Institute of Bioscience and Biotechnology, University of Science and Technology, Daejeon, Republic of Korea. ${ }^{5}$ These authors contributed equally: Su-Jin Park, Young-II Kim. *e-mail: jaeujung@med.usc.edu; choiki55@chungbuk.ac.kr 

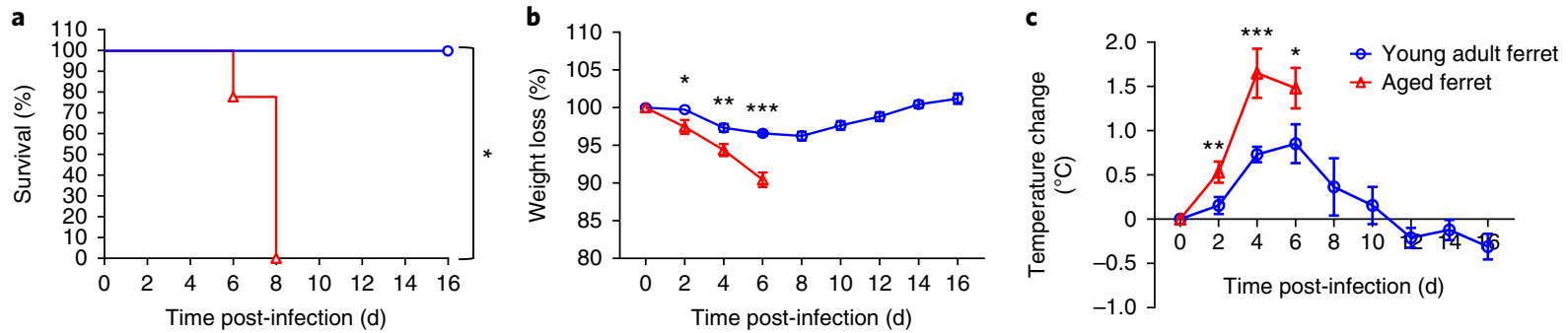

Fig. 1 | Survival and body weight of young adult and aged ferrets following inoculation with SFTSV. a-c, Nine ferrets in each group were i.m. inoculated with $10^{7.6} \mathrm{TCID}_{50}$ of virus. Survival (a), relative weight (b) and temperature (c) were assessed and are shown as standard error of the mean. Data (mean \pm s.e.m.) are presented in $\mathbf{b}$ and $\mathbf{c}$. The two-tailed Mantel-Cox method (a) or the two-tailed, unpaired $t$-test $(\mathbf{b}, \mathbf{c})$ was used to assess $P$ values. ${ }^{\star} P<0.0001$ (a), ${ }^{\star} P=0.0331 ;{ }^{\star \star} P=0.0069$ and ${ }^{\star \star \star} P<0.0001$ (b); and ${ }^{\star} P=0.0258,{ }^{\star \star} P=0.0062$ and ${ }^{\star \star \star} P<0.0709$ (c). The experiment was performed with three independent trials.
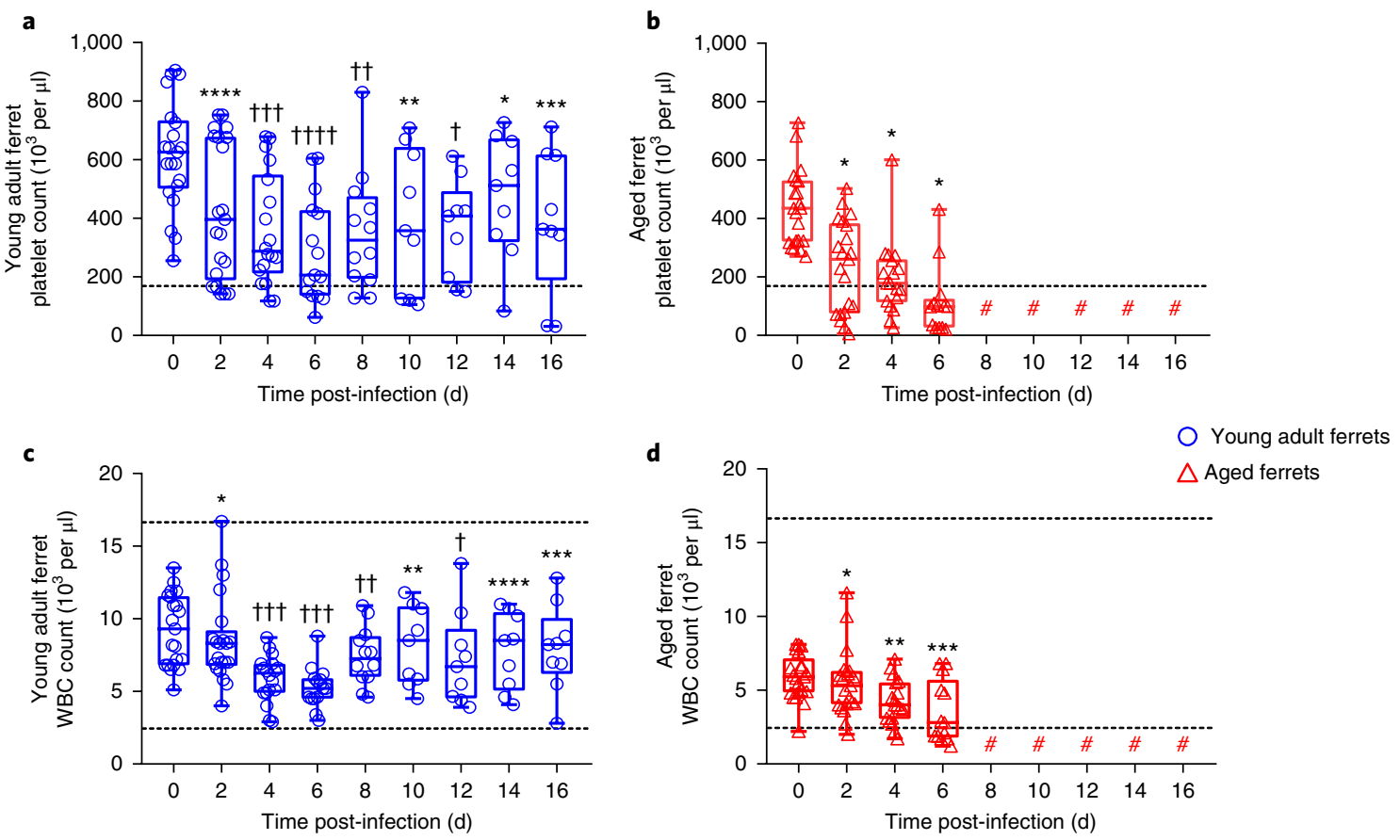

Fig. 2 Haematological analysis of SFTSV-inoculated ferrets ( $n=21$ per group). a-d, The blood from infected ferrets was collected every other day and haematological examination was performed using a Celltac hematology analyzer. Platelet counts from young adult ferrets (a), platelet counts from aged ferrets (b), WBC counts from young adult ferrets $(\mathbf{c})$ and WBC counts from aged ferrets $(\mathbf{d})$ are shown. Normal ranges of platelet and WBC counts in ferrets are 171.7-1,280.6 $\times 10^{3}$ per $\mu \mathrm{l}$ and $2.5-16.7 \times 10^{6}$ per $\mu$, respectively ${ }^{23,24}$. The dashed lines indicate the normal values of platelet or WBC counts. The sharp (\#) indicates no samples collected because the ferrets in this group died. Data are presented in box plots, with the box showing the upper (75\%) and lower (25\%) quartiles, the horizontal line as the median and the whiskers as the maximum and minimum values observed. The asterisks and daggers indicate significance between non-infected and infected ferrets per dpi as determined by a two-tailed unpaired $t$-test. ${ }^{\star} P=0.0783,{ }^{\star \star} P=0.0091$,

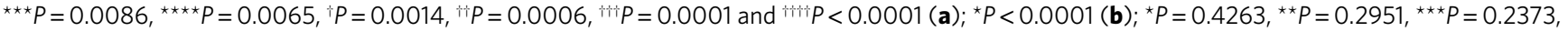
${ }^{\star \star \star \star} P=0.1614,{ }^{\dagger} P=0.0738,{ }^{\dagger} P=0.0397$ and ${ }^{\dagger} P<<0.0001(\mathbf{c})$; and ${ }^{\star} P=0.5176,{ }^{\star \star} P=0.0014$ and ${ }^{\star \star \star} P=0.0007$ (d).

Neither mice nor young adult ferrets exhibited any mortality over the course of the experiment even when inoculated with the highest titre of SFTSV CB1/2014 (Supplementary Fig. 1a,c). Furthermore, SFTSV-inoculated mice exhibited a $10-25 \%$ increase in mean body weight at 6-10 days post-infection (dpi) (Supplementary Fig. 1b). By contrast, there was a $4-5 \%$ decrease in the mean body weight of inoculated ferrets at 4-6 dpi with slightly increased body temperatures $\left(1.3^{\circ} \mathrm{C}\right)$ and a recovery of their original body weight by $8 \mathrm{dpi}$ (Supplementary Fig. 1d,e). These results demonstrate that young adult mice and ferret models do not exhibit severe clinical signs of SFTS, and hence may not be representative models for fatal SFTSV infection in humans.
Based on reported clinical symptoms and mortality rates, human patients over 50 years of age exhibited considerably severe SFTSV infection $^{22}$. Thus, we hypothesized that aged animals might display symptoms reflective of human infection with SFTSV. We administered the SFTSV CB1/2014 strain via an i.m. injection into aged mice of each strain ( $\geq 20$ months, BALB/c, C3H, C57BL/6 and FVB; $n=5)$ and into aged ferrets ( $\geq 4$ years of age, $n=5)$ and then compared the clinical manifestations with those of young adult mice and ferrets, respectively. All inoculated mice survived the SFTSV infection, although they showed a slight weight loss at 2-6dpi before it recovered at 8-16 dpi (Supplementary Fig. 2a,b). By marked contrast, 4 out of 5 aged ferrets (80\%) exhibited decreased body weight 

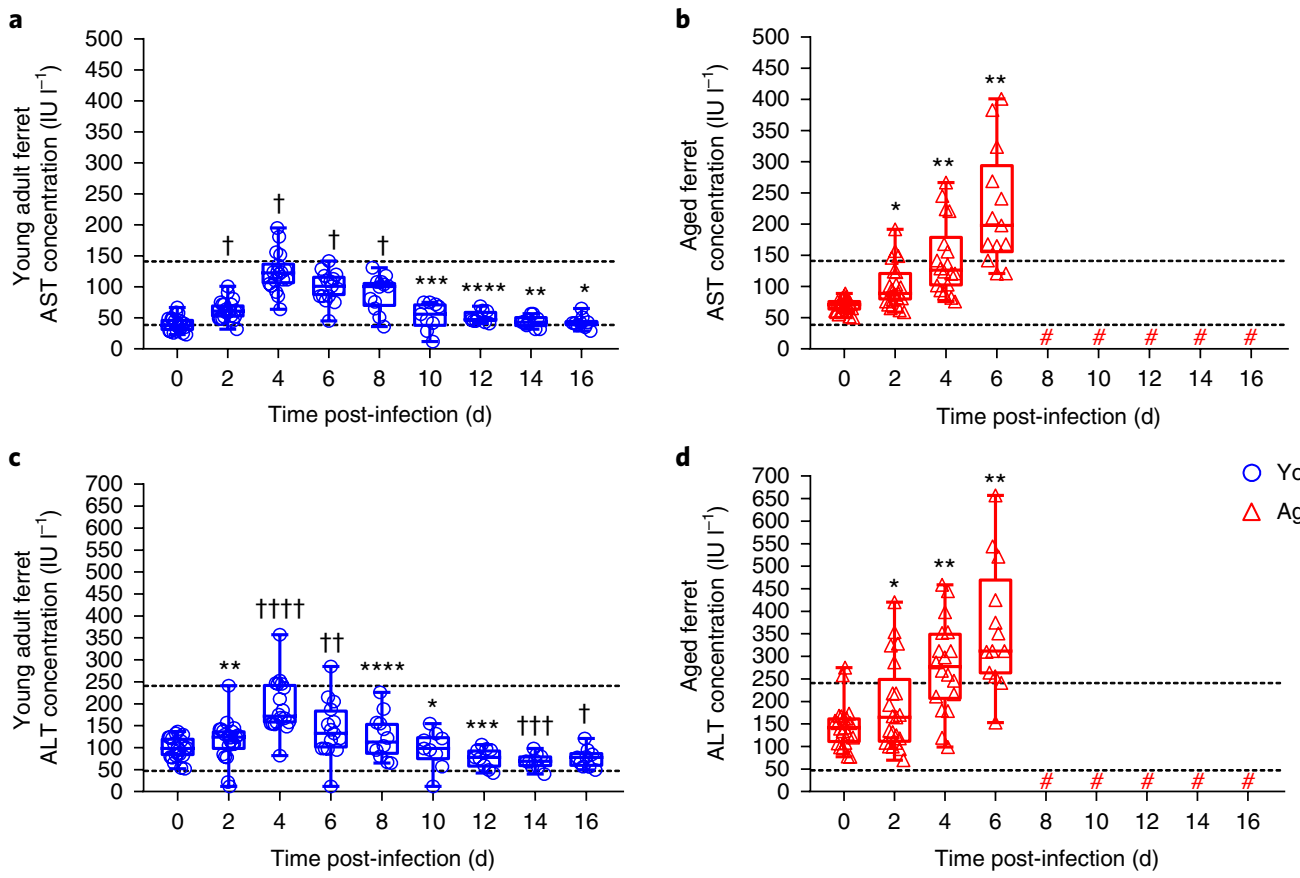

Fig. 3 | Concentration of liver enzymes in the blood of SFTSV-inoculated ferrets ( $\boldsymbol{n}=\mathbf{2 1}$ per group). a-d, The blood from infected ferrets was collected every other day and haematological examination was performed using a Celltac hematology analyzer. AST concentrations in young adult ferrets (a), AST concentrations in aged ferrets (b), ALT concentrations in young adult ferrets (c) and ALT concentrations in aged ferrets (d) are shown. The normal range of AST or ALT concentration is marked by the dashed lines in each panel. The sharp (\#) indicates no samples collected because the ferrets in this group died. Data are presented in box plots, with the box showing the upper (75\%) and lower (25\%) quartiles, the horizontal line as the median and the whiskers as the maximum and minimum values observed. The asterisks or daggers indicate significance between non-infected and infected ferrets per dpi as determined by a two-tailed, unpaired $t$-test. ${ }^{\star} P=0.5246,{ }^{\star \star} P=0.3488,{ }^{\star \star \star}{ }^{\star \star} P=0.0486,{ }^{\star \star \star \star} P=0.0174$ and ${ }^{\star} P<0.0001(\mathbf{a})$; ${ }^{\star} P=0.0003$ and

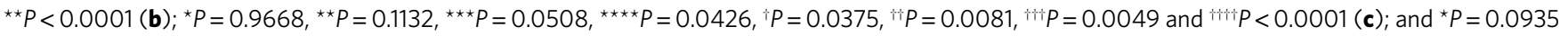
and ${ }^{\star \star} P<0.0001(\mathbf{d})$.

starting at 2 dpi (Supplementary Fig. 2d) and eventually succumbed to infection by $8 \mathrm{dpi}$ (Supplementary Fig. 2c). Moreover, body temperature of those that succumbed increased by $2.5^{\circ} \mathrm{C}$ at $2-6 \mathrm{dpi}$ before eventually dropping down below the normal range. Only one aged ferret returned to normal temperature at $8 \mathrm{dpi}$, recovered body weight at 12 dpi and ultimately survived (Supplementary Fig. 2c-e). Collectively, these results demonstrate that, although SFTSV infection has limited pathogenic effects on young or aged mice, SFTSVinfected aged ferrets exhibit severe clinical symptoms and a high mortality rate. Thus, we utilized ferrets as an animal model to evaluate the pathogenesis and clinical manifestation of SFTSV infection in an age-dependent manner.

Pathogenesis and clinical manifestation of SFTSV infection in ferrets. To compare the pathogenesis of SFTSV in young adult and aged ferrets, groups of 2-year-old young adult ferrets and 4-year-old aged ferrets were inoculated with $10^{7.6} \mathrm{TCID}_{50}$ per ml of the SFTSV $\mathrm{CB} 1 / 2014$ strain via an i.m. injection, and body weight and survival were monitored for 16 days. Although most natural infections are caused by tick bites (subdermal), we chose the i.m. route for virus administration, which showed similar results to subcutaneous delivery. As demonstrated in Fig. 1, no mortality was observed in young adult ferrets for 16 days (Fig. 1a) and they showed only a $5 \%$ decrease in body weight at $6 \mathrm{dpi}$ (Fig. 1b) with a mild increase in body temperature (Fig. 1c). However, all aged ferret groups showed severe clinical symptoms with high body temperatures and weight loss beginning at $2 \mathrm{dpi}$ and all aged ferrets succumbed by $8 \mathrm{dpi}$ (Fig. 1).

As thrombocytopenia is one of its major clinical symptoms, we collected blood from SFTSV CB1/2014-infected young adult and aged ferrets and compared their homeostasis factors. Our results showed that the mean platelet count of SFTSV-infected young adult ferrets was normal, although it fell to baseline at $6 \mathrm{dpi}$ and returned to the normal range $\left(171.7-1,280.6 \times 10^{3} \text { per } \mu \mathrm{l}\right)^{23,24}$ at $8 \mathrm{dpi}$ (Fig. 2a). By contrast, SFTSV-infected aged ferrets exhibited more rapid severe thrombocytopenia with a $235 \times 10^{3}$ per $\mu \mathrm{l}$ mean platelet count at $2 \mathrm{dpi}$ than young adult ferrets $\left(400 \times 10^{3}\right.$ per $\mu$ mean platelet count) $(P<0.0001)$, and their platelet numbers continuously decreased to below the normal range from $6 \mathrm{dpi}(P<0.0001)$ until death (Fig. 2b). We also assessed the number of white blood cells (WBCs), red blood cells and haemoglobin in SFTSV-infected young adult and aged ferrets. The mean WBC counts of SFTSV-infected young adult ferrets decreased until $6 \mathrm{dpi}$ and then recovered by $8 \mathrm{dpi}$, whereas a gradual decrease in WBC counts of SFTSV-infected aged ferrets continued until death (Fig. $2 \mathrm{c}, \mathrm{d}$ ). In addition, the mean numbers of red blood cells and the levels of haemoglobin of SFTSVinfected young adult and aged ferrets were still within their normal ranges $^{3,24}$ (Supplementary Fig. 3).

Similar to studies of SFTSV-infected humans ${ }^{25-27}$, blood biochemical analysis of infected ferrets showed elevated levels of aspartate aminotransferase (AST; also known as serum glutamic oxaloacetic transaminase) and alanine aminotransferase (ALT; also known as serum glutamic-pyruvic transaminase). The AST concentration of the SFTSV-infected young adult ferret group increased by about twofold at $4 \mathrm{dpi}$ and decreased thereafter (Fig. 3a), but their mean AST level $\left(117 \mathrm{Ul}^{-1}\right)$ was still within the normal range of 40.1-142.7 $\mathrm{Ul}^{-1}$ (ref. ${ }^{24}$ ). However, SFTSV-infected aged ferrets showed a gradual increase in the AST concentration until death, and the peak mean concentration was higher than $208 \mathrm{Ul}^{-1}$ (Fig. 3b). Although the ALT concentration (normal range: $\left.49-242.8 \mathrm{Ul}^{-1}\right)^{24}$ showed a similar pattern to AST in both ferret groups, the ALT concentration of SFTSV-infected 
a
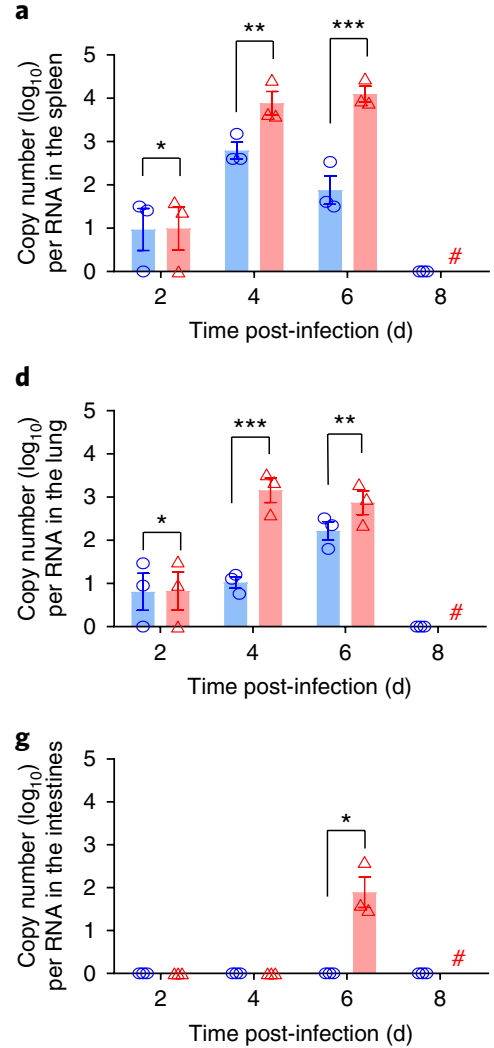

b

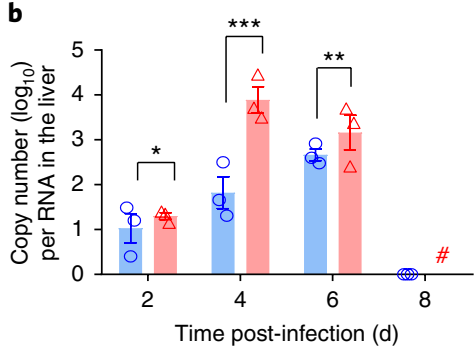

e

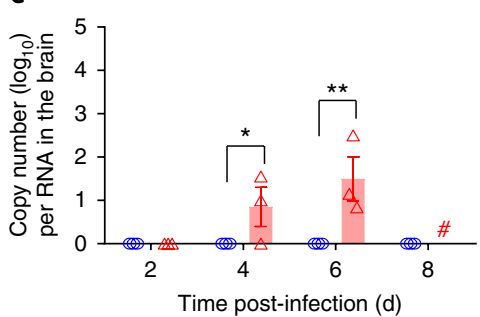

h

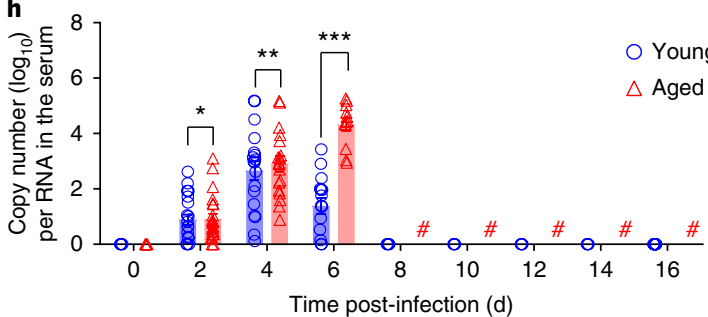

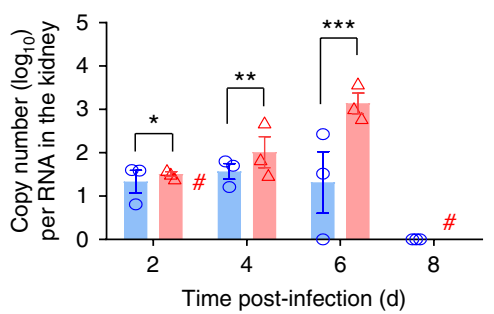

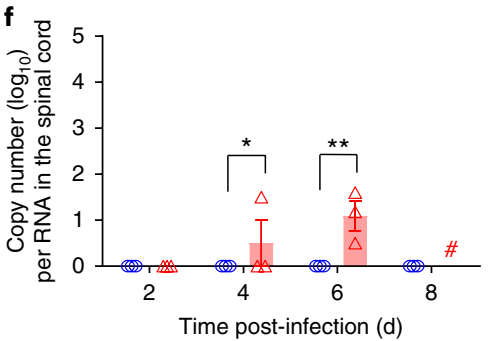

Young adult ferrets

Aged ferrets

Fig. 4 | Distribution of the number of viral RNA copies in tissues and blood of SFTSV-infected ferrets. a-h, Tissues ( $n=3$ per group) were collected at 2, 4, 6 and $8 \mathrm{dpi}$ and blood samples ( $n=21$ per group) were collected at $0,2,4,6,8,10,12,14$ or $16 \mathrm{dpi}$. The spleen (a), liver (b), kidney (c), lung (d), brain (e), spinal cord (f), intestines ( $(\mathbf{g})$ and serum $(\mathbf{h})$ were titred with real-time PCR. The sharp (\#) indicates no samples collected because the ferrets in this group died. Data are shown as mean \pm s.e.m. The asterisks indicate significance compared to each dpi sample by the two-tailed, unpaired $t$-test.

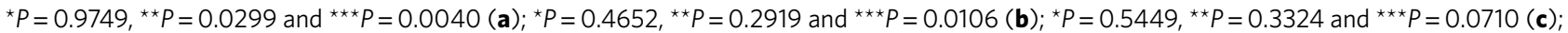
${ }^{\star} P=0.9757,{ }^{\star \star} P=0.1344$ and ${ }^{\star \star \star} P=0.0025(\mathbf{d}) ;{ }^{\star} P=0.1343$ and ${ }^{\star \star} P=0.0425(\mathbf{e}) ;{ }^{\star} P=0.3740$ and ${ }^{\star \star} P=0.0270(\mathbf{f}) ;{ }^{\star} P=0.0060(\mathbf{g})$; and ${ }^{\star} P=0.9170$, ${ }^{* *} P=0.5987$ and ${ }^{* \star *} P<0.0001(\mathbf{h})$. Each experiment was performed for three biological and three technical repeats.

aged ferrets reached a mean peak that was 1.7-times higher than that of SFTSV-infected young adult ferrets (Fig. 3c,d).

Taken together, the platelet and WBC counts and the AST and ALT concentrations of SFTSV-infected ferrets are important indicators of SFTSV pathogenesis, whereas the mean red blood cell counts and haemoglobin concentration do not seem to be critical readouts.

Viral distribution and replication in young adult and aged ferrets. Following infection in humans, SFTSV has been detected in multiple organs, indicating a systemic infection ${ }^{25,26}$. To identify similarity of SFTSV infection between ferrets and humans, the viral load in brain, lung, liver, spleen, kidney, intestine and spinal cord tissues was evaluated in infected ferrets. All organs of aged ferrets and the spleen, liver, kidneys and lungs of young adult ferrets were positive for SFTSV (Fig. 4). The spleen was the most susceptible organ, as it showed the highest viral load in both young adult and aged ferrets. Furthermore, in aged ferrets, the splenic viral load peaked at 6 dpi with $4.1 \log _{10}$ RNA copies per g, whereas in young adult ferrets, it peaked at $4 \mathrm{dpi}$ with $2.8 \log _{10}$ RNA copies per $\mathrm{g}$ followed by a gradual decrease until $8 \mathrm{dpi}$ (Fig. 4a). Viral load peaked at $4 \mathrm{dpi}$ in the liver $\left(3.9 \log _{10}\right.$ RNA copies per $\mathrm{g}$ ) of aged ferrets, then slightly decreased thereafter until death. In young adult ferrets, the viral load peaked in the liver at $6 \mathrm{dpi}$ with $2.7 \log _{10}$ RNA copies per $\mathrm{g}$ and then decreased dramatically at $8 \mathrm{dpi}$ (Fig. $4 \mathrm{~b}$ ), whereas in the kidney, a moderate virus load of $0.98-1.3 \log _{10}$ RNA copies per $g$ was observed and disappeared by 8 dpi. Even in the aged ferrets, a similar viral load was observed at 2 and $4 \mathrm{dpi}$ in the kidneys before reaching the peak at $6 \mathrm{dpi}$ with $3.1 \log _{10}$ RNA copies per $\mathrm{g}$ (Fig. $4 \mathrm{c}$ ). Furthermore, virus was also detected in the lungs with an increasing viral load starting at 2-6dpi in both young adult and aged ferrets. However, a higher load was detected in aged ferrets with a peak of $3.2 \log _{10}$ RNA copies per $g$ (than only $1.2 \log _{10}$ RNA copies per $g$ for young adult ferrets) at $4 \mathrm{dpi}$ (Fig. $4 \mathrm{~d}$ ). Furthermore, viral RNA was detected, albeit at relatively low copy numbers $\left(0.5-1.9 \log _{10}\right.$ RNA copies per g), in the brain, spinal cord and intestine only in the aged ferret group (Fig. 4e-g). In addition, SFTSV was isolated from most tissue specimens taken from aged ferrets, whereas only spleen tissues ( 2 out of 3 ) were positive for virus isolation in young adult ferrets (Supplementary Fig. 5).

Next, we assessed the correlation between the viral load in the blood of SFTSV-infected ferrets and disease pathogenesis and progress. Virus was first detected in the serum of both young adult and aged ferrets at $2 \mathrm{dpi}$ (Fig. $4 \mathrm{~h}$ ). In young adult ferrets, $0.75 \log _{10}$ viral copies per $0.1 \mathrm{ml}$ was detected at $2 \mathrm{dpi}$ and reached a peak of $2.7 \log _{10}$ viral copies per $0.1 \mathrm{ml}$ at $4 \mathrm{dpi}$. However, the viral load in sera was significantly decreased at $6 \mathrm{dpi}$ and then was not detected from $8 \mathrm{dpi}$ onwards (Fig. 4h). Although a low mean viral load $\left(0.9 \log _{10}\right.$ viral copies per $0.1 \mathrm{ml}$ ) was detected in aged ferrets at $2 \mathrm{dpi}$, the viral load increased continuously until the peak reached $4.3 \log _{10}$ viral copies per $0.1 \mathrm{ml}$ at $6 \mathrm{dpi}$, before a majority succumbed at $8 \mathrm{dpi}$ (Fig. $4 \mathrm{~h}$ ). This result clearly demonstrates that the viral load in the blood is strongly associated with the mortality of SFTSV-infected ferrets.

To confirm viral replication in the organs of infected ferrets, immunohistochemistry and RNAscope in situ hybridization assays 

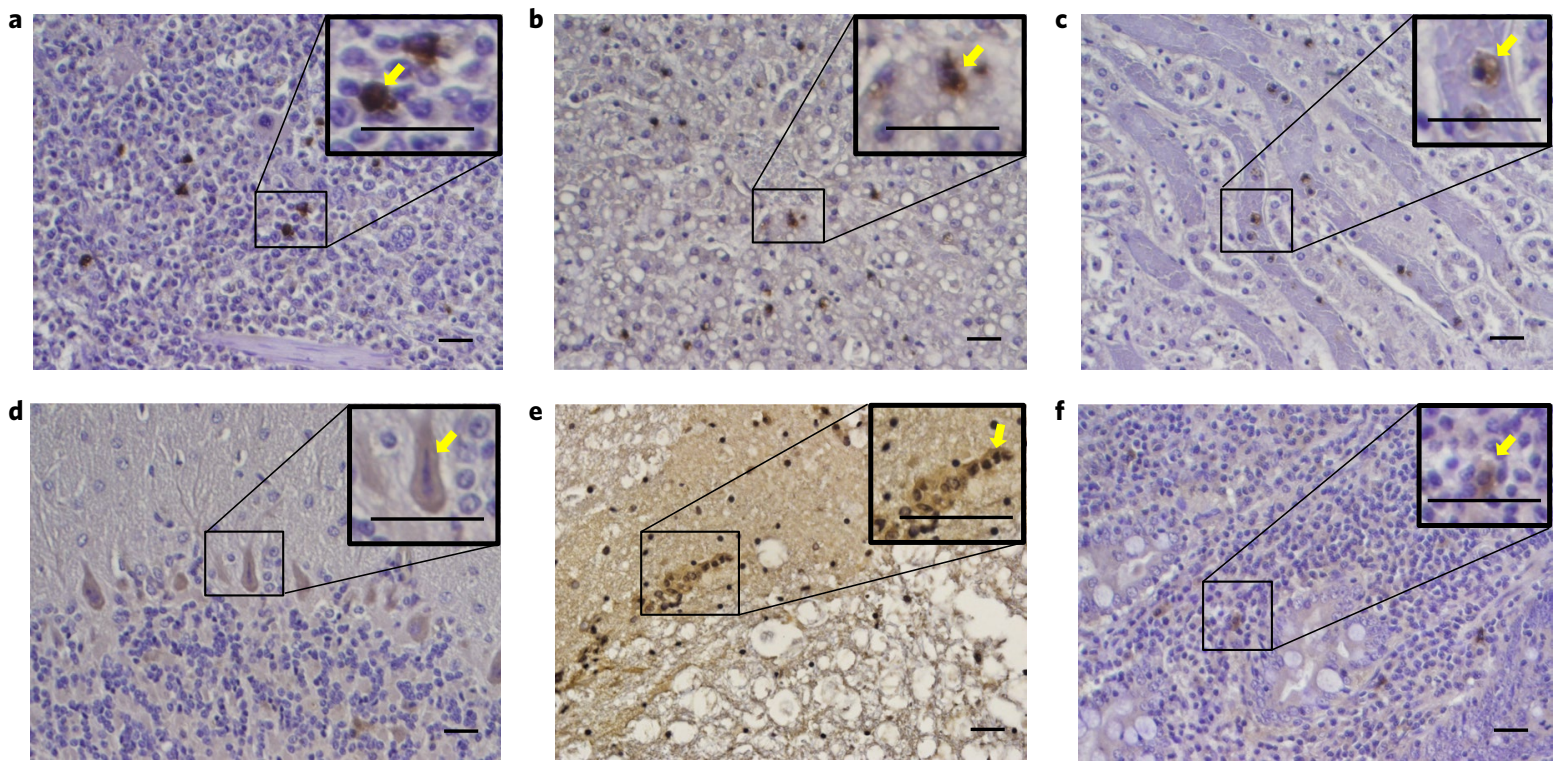

Fig. 5 | Immunohistochemistry of tissues from aged ferret infected with SFTSV. a-f, Ferrets were i.m. inoculated with $10^{7.6}$ TCID $_{50}$. Tissues were harvested on day 6 after inoculation. The presence of the SFTSV NP antigen was confirmed in the spleen (red pulp) (a), liver (central vein) (b), kidney (renal tubule) (c), brain (cerebellar cortex) (d), spinal cord (ventral horn) (e) and intestine (small intestinal crypts) (f) by immunohistochemistry. Magnification $\times 400$. SFTSV-positive regions were magnified $\times 400$ for each figure. Yellow arrows indicate SFTSV antigen-positive cells. Experiments were performed with three independent trials and similar results were reproduced. Scale bars, $20 \mu \mathrm{m}$.

to detect the SFTSV nucleoprotein (NP) and RNA, respectively, were conducted (Fig. 5 and Supplementary Figs. 6 and 7). These tests showed that SFTSV replication was readily detected in the spleen, liver, kidneys, brain, spinal cord and intestines of aged ferrets (Fig. 5 and Supplementary Fig. 7). In agreement with the viral load, SFTSV NP was also detected in the spleen, liver and kidneys (Supplementary Fig. 6a-c) of young adult ferrets. However, SFTSV NP-positive cells were only faintly detected in the brain, spinal cord and intestines (Supplementary Fig. 6d-f) of young adult ferrets. Furthermore, more severe histopathological damages were demonstrated in aged ferrets than in young adult ferrets (Supplementary Fig. 8). These findings clearly demonstrate that SFTSV can infect and replicate in multiple organs and suggest that systemic infection of ferrets occurs in an age-dependent manner.

Transcriptional profile of immune-related genes in peripheral blood mononuclear cells of SFTSV-infected ferrets. To compare global and dynamic gene expression in SFTSV-infected young adult and aged ferrets, RNA sequencing (RNA-seq) analysis was performed on peripheral blood mononuclear cells (PBMCs) from SFTSV-infected young adult and aged ferrets at 2 and $4 \mathrm{dpi}$. Using the 24,417 annotated genes in the ferret genome database, gene expression was quantified in both ferret groups and compared to that of the phosphate-buffered saline (PBS)-treated ferret control. Differentially expressed genes (DEGs) with a $P<0.05$ were identified, as previously described ${ }^{27}$. The differentially expressed mRNAs in SFTSV-infected young adult and aged ferrets (with \pm 2 -fold changes) are depicted as overlapping circles (Fig. 6a), with the number of differing mRNAs indicated in each area. Briefly, when compared to PBS-treated ferrets, a total of 539 genes were significantly changed in SFTSV-infected ferrets at all time points, regardless of age. Of these, 269 genes were upregulated and 268 genes were downregulated upon infection. The remaining two genes were not consistently expressed across time points. Among the immune-associated DEGs, 2'-5'-oligoadenylate synthetase-like (OASL), which has antiviral activity, was the most significantly upregulated gene followed by the C-X-C motif chemokine 10 (CXCL10), a well-known player in the antiviral response. The expression of these two genes was higher in the aged group than in the young adult group at $4 \mathrm{dpi}$ (54.80 versus 30.51 - and 36.22 versus 28.37 -fold change, respectively) (Fig. 6a). By contrast, the levels of interleukin-5 (IL-5) receptor- $\alpha$ and IL-20 were significantly downregulated. Expression patterns of the upregulated OASL and CXCL10 genes and the downregulated IL5RA and IL20 genes were confirmed by real-time reverse transcription (RT)-PCRs (Supplementary Fig. 9).

To investigate possible biological interactions of DEGs and identify important functional networks, we imported data sets representing genes with altered expression profiles derived from the RNA-seq analyses into the Ingenuity Pathway Analysis (IPA) tool. The highest activated networks (high $\mathrm{z}$-score) were then identified using IPA. The heat map shows the canonical pathways activated by SFTSV infection in young adult and aged ferrets compared to the PBS control group (Fig. 6b). Notably, many key innate immune response pathways, including RIG-I virus sensing, IFN signalling and IFN regulatory factors (IRFs), were significantly upregulated in all SFTSV-infected groups compared to the PBS control group. Interestingly, there was a decrease in IFN and IRF signalling from 2 to $4 \mathrm{dpi}$ in young adults ( $\mathrm{z}$-score: 1.51 versus 1.0 and 1.96 versus 0.0 , respectively), whereas the aged group showed an increase (Fig. 6b). Furthermore, many of the signalling pathways (that is, the dendritic cell maturation pathway and the p38 mitogen-activated protein kinase signalling pathway) that were upregulated at 2 dpi were subsequently downregulated at $4 \mathrm{dpi}$ in the young adult ferret group, reflecting their recovery from SFTSV infection.

Given the different clinical outcomes of young adult and aged ferrets upon SFTSV infection, we further investigated the expression of other critical DEGs between two groups (Fig. 6c). Many inflammatory-associated pathways, such as dendritic cell maturation (z-score: 2.79), leukocyte extravasation (z-score: 2.65 ) and IL-6 signalling (z-score: 1.94$)$, were significantly upregulated in aged ferrets compared to the young adult ferrets, suggesting severe inflammatory responses in older ferrets. By contrast, the apoptosis-associated signalling pathway was downregulated in aged ferrets. Furthermore, higher levels of inflammatory macrophage recruitment factors, such 


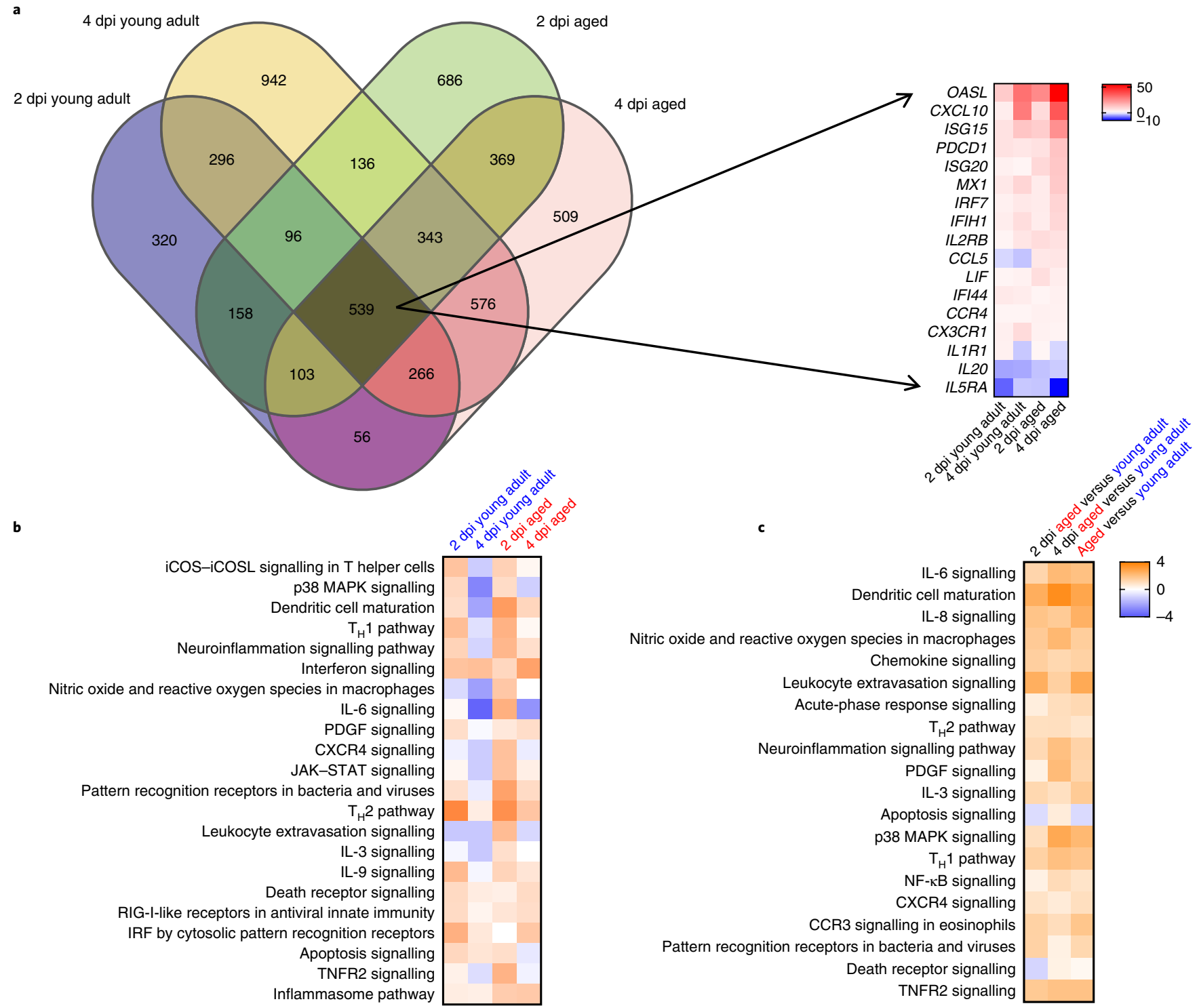

Fig. 6 | Overview of RNA-seq data from PBMCs of young adult and aged ferrets infected with SFTSV and control ferrets at 2 and $4 \mathrm{dpi}(n=\mathbf{2}$ for control and $\mathbf{2} \mathrm{dpi}$ of young adult ferrets and $\boldsymbol{n}=\mathbf{3}$ for $\mathbf{4} \mathrm{dpi}$ of young adult ferrets and aged ferrets). Comparison of DEGs identified immune-related genes as shown by Venn diagrams and heat maps. a, Genes upregulated or downregulated by more than twofold were selected from among mock and young adult or aged ferret groups (DEGs were identified with Bonferroni-corrected $P$ value, $P<0.05$ ). $\mathbf{b}$, Canonical signalling pathways activated by SFTSV infection. The IPA tool was used to generate a list of the significant canonical pathways and the activated networks with their respective scores. A comparison of gene expression among mock and young adult or aged ferret groups is shown. c, Canonical signalling pathways activated by SFTSV infection. A comparison of gene expression between aged and young adult ferrets is shown. The RNA-seq data were statistically analysed by the two-sided Wald test (FDR < 0.05). CCR3, C-C chemokine receptor type 3; CXCR4, C-X-C chemokine receptor type 4; iCOS, inducible T cell costimulator, iCOSL, iCOS ligand; JAK, Janus kinase; MAPK, mitogen-activated protein kinase; NF- $\mathrm{\kappa B}$; nuclear factor- $\mathrm{\kappa B}$; PDGF, platelet-derived growth factor; STAT, signal transducer and activator of transcription; $T_{H}$, T helper; TNFR2, tumour necrosis factor receptor superfamily member 2.

as transforming growth factor- $\beta 2$ and matrix metalloproteinase 9 , were present in aged ferrets than in young adult ferrets, which implied increased inflammatory cell infiltration into sites of infection in aged ferrets (Supplementary Fig. 10).

\section{Discussion}

Despite the increasing incidence of SFTSV human infections and the public health concerns caused by this virus, details regarding the pathogenesis of this virus are largely unknown due to the lack of a proper experimental model. In this study, we demonstrate the age-associated mortality of SFTSV infection using a ferret model. Although the case fatality rates (CFR) in human patients varied from 5.3 to $27 \%$ depending on which year and which country reports were from ${ }^{7,9-12}$, aged ferrets showed a CFR as high as $93 \%$. The underlying reason for this marked difference might be explained by two factors, the first being that the dose $\left(10^{7.6} \mathrm{TCID}_{50}\right.$ per $\mathrm{ml}$ ) of SFTSV introduced into the animal model is probably much higher than that introduced to humans by a tick bite. The second reason may be age. Although SFTSV infection has fatality rates ranging from $5 \%$ to as high as $30 \%$ in all ages of infected human, it exhibits a drastically increased fatality rate in human patients who are more than 50 years of age $\mathrm{e}^{7,9-12}$. Similarly, no case mortality was observed in young adult ferrets and a high CFR following SFTSV infection was observed in aged ferrets. Thus, the 
high CFR in this study may be due to a synergistic effect of high inoculum dose and age. The results of haematological analysis clearly recapitulated the clinical manifestation of fatal human disease (Figs. 2 and 3). According to a meta-analysis of human patients with SFTSV infection in China, older and immunocompromised patients had difficulty recovering platelet and WBC counts, which was believed to lead to high mortality ${ }^{19}$. This finding is well within agreement with our aged ferret experiment. Furthermore, the aged ferrets showed significantly increased AST and ALT concentrations up until death, suggestive of viral hepatitis as was reported in human patients ${ }^{26}$. These haematology results clearly demonstrate that haemostasis in SFTSV-infected aged ferrets is reflective of that seen in severe-to-fatal human cases of SFTSV infection ${ }^{8}$. It should be noted that, within the scope of animal model development for SFTSV infection and pathogenesis, we did not determine the specific 50\% lethal dosage in ferrets, as ferrets are outbred animals unlike inbred mice and, specially, aged ferrets are quite limited. However, it would be important for future works, such as the evaluation of therapeutic drugs or vaccines.

SFTSV was frequently detected in the serum, spleen, lungs, liver and kidneys both in young adult and in aged ferrets (Fig. 4). In addition, histopathological staining with SFTSV antibody revealed a high viral load especially in the spleen, liver and kidneys in aged ferrets. Although young adult and aged ferrets showed virus replication in most of the peripheral organs (the spleen, liver, kidneys, lungs and blood), only aged ferrets showed viral transcripts in the brain and spinal cord. These results also suggest that the dissemination of SFTSV into the central nervous system might be associated with the death of aged ferrets. However, the dissemination of SFTSV in the brain and spinal cord was not a unique feature of the aged ferrets as the detection of SFTSV in the brain and spinal cord has been previously reported in knockout mouse models and human infection cases $^{16,28,29}$. Although no central nervous system-related symptoms were observed in the current study, possibly due to the fact that infected ferrets succumbed rapidly, further study will be required to elucidate any association between SFTSV dissemination into the central nervous system and case fatality. It should be noted that, although the virus could be transiently detected in the spleen, liver and kidneys of infected aged mice, the virus copy number was significantly lower than that seen in infected ferrets (Fig. 4 and Supplementary Fig. 4). With this attenuated virus replication, the immune-competent mouse strains did not induce any clinical symptoms and, hence, would not be a useful model for SFTSV-induced diseases in humans.

Many factors differentiate the degree of disease caused by this SFTSV between young adult and aged ferrets. Of these, the immune response seems to be a critical factor for protection from this virus. Transcriptional profile analyses of PBMCs revealed that IFN and IRF signalling pathways were significantly upregulated in SFTSVinfected groups compared to the PBS control group, although higher $\mathrm{Z}$-scores were observed in young adult ferrets than in aged ferrets (Fig. 6b). Further analysis demonstrated that many inflammatoryassociated pathways were significantly upregulated and persisted in aged ferrets compared to the young adult ferrets. For instance, CCL5 chemokine, which plays an active role in recruiting $\mathrm{T}$ cells, eosinophils and basophils into inflammatory sites, was persistently upregulated only in aged ferrets (Fig. 6a and Supplementary Fig. 9e). Thus, this suggests that severe and persistent inflammatory responses are mediated by activated immune-modulating cells in aged ferrets. This correlates with the high viral load in the aged group (Figs. 4 and $6 \mathrm{c}$ ). Together, these results suggest that the rapid and strong responses of IFN and IRF signalling pathways effectively suppress early virus infection and proliferation, eventually leading to rapid clearance of SFTSV from young adult ferrets. By contrast, aged ferrets exhibit delayed IFN and IRF responses with persistently upregulated inflammatory immune responses, such as the production of chemokines associated with leukocyte extravasation, and a high production of nitric oxide and reactive oxygen species by macrophages (z-score: 1.54), resulting in tissue damage and mortality of SFTSV-infected aged ferrets. Both aged humans and ferrets may have attenuated immune function that is necessary to protect the body against invading antigens. This is thought to be due to decreases in the number of plasma fluid cells and monocytes ${ }^{30,31}$. Thus, an attenuated immune system seems to underlie the marked replication of viral pathogens, such as SFTSV, in aged populations, consequently causing increased disease severity and eventually leading to death.

Taken together, our data indicate that at least three different factors may be involved in SFTSV-induced mortality: the depletion of platelets and WBCs, delayed innate immune responses and a high viral load in the bloodstream. Furthermore, our results suggest the potential usefulness of ferrets as an animal model for SFTSV pathogenesis and that the ferret model would be suitable for the assessment of many biomedical parameters, such as antiviral drug screening and vaccine efficacy tests.

\section{Methods}

Virus and cells. SFTSV strain CB1/2014 was isolated from the sera of SFTSVinfected patients who were hospitalized with typical SFTS symptoms at Chungbuk National University Hospital (Cheongju, South Korea) ${ }^{21}$. For virus propagation, the virus was passaged five times on confluent monolayers of Vero E6 cells (ATCC no. CRL-1586; American Type Culture Collection) in DMEM (Gibco) containing $2 \%$ FBS (Gibco) with penicillin $\left(100 \mathrm{U} \mathrm{ml}^{-1}\right)$ and streptomycin $\left(100 \mu \mathrm{g} \mathrm{ml}^{-1}\right.$; Gibco $)$ placed in a $37^{\circ} \mathrm{C}$ incubator supplemented with $5 \% \mathrm{CO}_{2}$. Cell culture supernatant was collected at $7 \mathrm{dpi}$ and stored at $-80^{\circ} \mathrm{C}$ as the working virus stock for animal studies. Viral infectivity titres were determined through an immunostaining assay in which the TCID $_{50}$ was determined with an in-house-generated monoclonal NP antibody against SFTSV in an immunofluorescence assay.

Mouse infection study. Eight-week-old or aged ( $\geq 20$ months of age) mice ( $n=17$ per group) of each strain (BALB/c, C3H, C57BL/6 and FVB) were i.m. inoculated with $10^{6.6} \mathrm{TCID}_{50}$ per animal of SFTSV CB1/2014 strain $(50 \mu \mathrm{l}$ in the outside of the thigh of both legs). The mice ( $n=5$ per group) were monitored for body weight and clinical symptoms daily for 16 days. The remaining mice ( $n=3$ per group) were killed to evaluate the viral copy numbers in tissues at 2, 4, 6 and $8 \mathrm{dpi}$.

Ferret infection study. Two groups of young adults (20-24 months of age, $\leq 2$ years of age) and aged ferrets (48-50 months of age), $\geq 4$ years of age) were inoculated via the i.m. route with $10^{7.6} \mathrm{TCID}_{50}$ of the SFTSV CB1/2014 strain $(0.5 \mathrm{ml}$ in the outside of the thighs of both legs). Blood was collected from anaesthetized ferrets every other day for various assays and virus titration. To access virus replication of SFTSV-infected ferrets, a single ferret from each group was killed at 2, 4, 6 or $8 \mathrm{dpi}$, and the brain, lung, liver, spleen, kidney, intestine and spinal cord tissues were collected with individual scissors to avoid the cross-contamination. This study was conducted with three independent trials; hence, a total of 21 ferrets were infected in each group. In this study, two trials ( 14 ferrets) used female ferrets and one trial was conducted with 7 male ferrets. However, the CFR and haematological parameters were similar between sexes.

Haematological and biochemical assays. Haematological parameters were analysed in EDTA-treated whole blood from infected animals using the Celltac hematology analyzer (MEK-6550J/K, Nihon Kohden). Biochemical parameters of serum from infected animals were determined using Celltac- $\alpha$ (MEK-6550, Nihon Kohden)

Real-time PCR. Total RNA was extracted using TRIzol reagent (Thermo Fisher Scientific) or the RNeasy kit (Qiagen) and cDNAs were generated by reverse transcription using QuantiTect Reverse Transcription (Qiagen). Viral copy numbers were determined by real-time RT-PCR with an M segment-based SFTSV-specific primer set. The forward primer was SFTSV-M-F: AATTCACATTTGAGGGTAGTT and the reverse primer was SFTSV-M-R: TATCCAAGGAGGATGACAATAAT Copy numbers were calculated as a ratio with respect to the standard control ${ }^{28}$, and gene expression of relative fold change was calculated with ${ }^{\Delta \Delta} \mathrm{CT}$ methods and normalized to the expression levels of the GAPDH gene. Real-time PCR reactions were performed using a SYBR Green Supermix (Bio-Rad) and a CFX96 Touch RealTime PCR Detection System (Bio-Rad).

Immunohistochemistry. For immunohistochemistry analysis, we collected tissue samples (the brain, lung, liver, spleen, kidney, intestine and spinal cord) at $6 \mathrm{dpi}$ 
from CB1/2014-infected young adult or aged ferrets. The samples were fixed in $10 \%$ neutral-buffered formalin and embedded in paraffin according to the standard procedures. The embedded tissues were sectioned and dried for 3 days at room temperature. To detect the viral antigen by immunohistochemistry, a monoclonal antibody against SFTSV was used as the primary antibody. Antigen was visualized using the biotin-avidin system (Vector Labs). Slides were viewed using the Olympus IX 71 (Olympus) microscope with DP controller software to capture images.

RNAscope in situ hybridization. SFTSV RNA (NP segment) was detected using the NP-specific probe (552071, Advanced Cell Diagnotics) and visualized using the RNAscope 2.5 HD Reagent Kit RED (322360, Advanced Cell Diagnotics). Organ tissue sections were fixed in 10\% neutral-buffered formalin and embedded in paraffin, according to the manufacturer's instructions, followed by counterstaining with haematoxylin (H3401, Vector Laboratories). Sections were imaged using the BZ 9000 all-in-one Fluorescence Microscope from Keyence and analysed using the NIH Image J software.

PBMC isolation. PBMCs were isolated at 2 and 4 dpi from each group of ferrets $(n=3)$ by density gradient centrifugation using Ficoll-Paque Plus (GE Healthcare Biosciences) according to the manufacturer's protocol. Total RNA was extracted from PBMCs of mock PBS control and SFTSV CB1/2014-infected ferrets using the RNeasy Micro Kit (Qiagen) according to the manufacturer's protocol. RNA quantity was assessed by spectrophotometry using Nanodrop 1000 (Thermo Fisher Scientific)

Analysis of the RNA-seq transcriptome. To evaluate the differential expression of cellular response in PBMCs, RNA-seq was performed. The cDNA libraries were constructed from PBMC RNA samples and was sequenced using an Illumina HiSeq 2500 sequencer, as described previously. One paired-end library with an insert size for each sample of $100 \mathrm{bp}$ was sequenced. Raw reads were assembled using CLC Genomics Workbench (version 11), in which low-quality reads were filtered and the filtered reads were subsequently mapped to a reference genome downloaded from Ensembl (MusPutFur1.0). Data analysis was performed using the two-sided Wald test to calculate false discovery rates (FDR-adjusted $P<0.05$ ) and to identify DEGs. Furthermore, DEGs generated molecular networks according to biological and molecular importance, including canonical pathways, upstream and downstream regulatory analysis through the Ingenuity Knowledge Base. Fisher's exact test $(P>0.05)$ and inhibition or activation $\mathrm{z}$-scores were calculated by IPA.

Statistical analysis. The asterisks indicate significant differences in weight loss, temperature change, blood analysis, viral load and titre between groups and across time points as determined by the two-tailed Mantel-Cox method or two-tailed unpaired $t$-test. Statistical analyses were performed using GraphPad Prism version 8.00 for Windows (GraphPad Software)

Ethics statement. All animal experiments were approved by the Medical Research Institute, a member of the Laboratory Animal Research Center of Chungbuk National University (LARC) (approval number: CBNUA-986-16-01), and were conducted in strict accordance and adherence to relevant policies regarding animal handling as mandated under the Guidelines for Animal Use and Care of the Korea Centers for Disease Control and Prevention. Viruses were handled in an enhanced biosafety level 3 containment laboratory as approved by the Korean Centers for Disease Control and Prevention (KCDC-14-3-07).

Reporting Summary. Further information on research design is available in the Nature Research Reporting Summary linked to this article.

\section{Data availability}

The data that support the findings of this study are available from the corresponding authors upon request. The RNA-seq data were deposited in the Gene Expression Omnibus (GEO) under the accession number GSE121911.

Received: 19 May 2018; Accepted: 6 November 2018; Published online: 10 December 2018

\section{References}

1. Wiwanitkit, S. \& Wiwanitkit, V. Acute viral hemorrhage disease: a summary on new viruses. J. Acute Dis. 4, 277-279 (2015).

2. Adams, M. J. et al. Changes to taxonomy and the International Code of Virus Classification and Nomenclature ratified by the International Committee on Taxonomy of Viruses (2017). Arch. Virol. 162, 2505-2538 (2017).

3. Kim, K.-H. et al. Severe fever with thrombocytopenia syndrome, South Korea, 2012. Emerg. Infect. Dis. 19, 1892-1894 (2013).

4. Takahashi, T. et al. The first identification and retrospective study of severe fever with thrombocytopenia syndrome in Japan. J. Infect. Dis. 209, 816-827 (2013).
5. SFTSV Infection in Korea (KCDC, 2016); http://cdc.go.kr/CDC/mobile/notice/ CdcKrIntro0201.jsp? menuIds=HOME001-MNU1154-MN\&fid=21\&q_type $=$ \&q_value $=\&$ cid $=73941$ \&pageNum $=1$

6. NIID. Severe fever with thrombocytopenia syndrome (SFTS) in Japan, as of February 2016. IASR 37, 39-40 (2016).

7. Zhan, J. et al. Current status of severe fever with thrombocytopenia syndrome in China. Virol. Sin. 32, 51-62 (2017).

8. $\mathrm{Li}, \mathrm{D}$. Severe fever with thrombocytopenia syndrome: a newly discovered emerging infectious disease. Clin. Microbiol. Infect. 21, 614-620 (2015).

9. Baba, M. et al. Establishment of an antiviral assay system and identification of severe fever with thrombocytopenia syndrome virus inhibitors. Antivir. Chem. Chemother. 25, 83-89 (2017).

10. Disease Web Statistics System (KCDC, 2017); http://www.cdc.go.kr/npt/biz/ npp/ist/bass/bassDissStatsMain.do

11. Epidemiologic and Clinical Characteristics of Severe Fever with Thrombocytopenia Syndrome in the Republic of Korea (KCDC, 2016); http:// cdc.go.kr/CDC/together/CdcKrTogether0302.jsp?menuIds=HOME001MNU1154-MNU0005- MNU0088\&fid=51\&q_type=title\&q_value= SFTS\&cid $=69842 \&$ pageNum $=$

12. Caution of SFTS Infection at Outdoor Activity (KCDC, 2018); http://www.cdc. go.kr/npt/biz/npp/portal/nppSumryMain.do?icdCd=D0018\&icdgrpCd= 04\&icdSubgrpCd=

13. 2018 Annual Review of Diseases Prioritized Under the Research and Development Blueprint. WHO Research and Development Blueprint (WHO, 2018).

14. Pepin, M., Bouloy, M., Bird, B. H., Kemp, A. \& Paweska, J. Rift Valley fever virus (Bunyaviridae: Phlebovirus): an update on pathogenesis, molecular epidemiology, vectors, diagnostics and prevention. Vet. Res. 41, 61 (2010).

15. Swei, A. et al. The genome sequence of Lone Star virus, a highly divergent bunyavirus found in the Amblyomma americanum tick. PLoS ONE 8, e62083 (2013).

16. Liu, Y. et al. The pathogenesis of severe fever with thrombocytopenia syndrome virus infection in alpha/beta interferon knockout mice: insights into the pathologic mechanisms of a new viral hemorrhagic fever. J. Virol. 88, 1781-1786 (2014).

17. Chen, X.-P. et al. Infection and pathogenesis of Huaiyangshan virus (a novel tick-borne bunyavirus) in laboratory rodents. J. Gen. Virol. 93, 1288-1293 (2012).

18. Jin, C. et al. Pathogenesis of emerging severe fever with thrombocytopenia syndrome virus in C57/BL6 mouse model. Proc. Natl Acad. Sci. USA 109, 10053-10058 (2012).

19. Liu, S. et al. Systematic review of severe fever with thrombocytopenia syndrome: virology, epidemiology, and clinical characteristics. Rev. Med. Virol. 24, 90-102 (2014).

20. Jin, C. et al. SFTS virus infection in nonhuman primates. J. Infect. Dis. 211, 915-925 (2014).

21. Yun, S.-M. et al. Molecular genomic characterization of tick-and humanderived severe fever with thrombocytopenia syndrome virus isolates from South Korea. PLoS Negl. Trop. Dis. 11, e0005893 (2017).

22. Sun, J. et al. Factors associated with severe fever with thrombocytopenia syndrome infection and fatal outcome. Sci. Rep. 6, 33175 (2016).

23. Smith, S. A., Zimmerman, K. \& Moore, D. M. Hematology of the domestic ferret (Mustela putorius furo). Vet. Clin. North Am. Exot. Anim. Prac. 18, 1-8 (2015).

24. Hein, J., Spreyer, F., Sauter-Louis, C. \& Hartmann, K. Reference ranges for laboratory parameters in ferrets. Vet. Rec. 171, 218 (2012).

25. Li, S. et al. Multiple organ involvement in severe fever with thrombocytopenia syndrome: an immunohistochemical finding in a fatal case. Virol. J. 15, 97 (2018)

26. Hiraki, T. et al. Two autopsy cases of severe fever with thrombocytopenia syndrome (SFTS) in Japan: a pathognomonic histological feature and unique complication of SFTS. Pathol. Int. 64, 569-575 (2014).

27. Park, S.-J. et al. Dynamic changes in host gene expression associated with $\mathrm{H} 5 \mathrm{~N} 8$ avian influenza virus infection in mice. Sci. Rep. 5, 16512 (2015).

28. Shimada, S., Posadas-Herrera, G., Aoki, K., Morita, K. \& Hayasaka, D. Therapeutic effect of post-exposure treatment with antiserum on severe fever with thrombocytopenia syndrome (SFTS) in a mouse model of SFTS virus infection. Virology 482, 19-27 (2015).

29. Kaneko, M. et al. A patient with severe fever with thrombocytopenia syndrome and hemophagocytic lymphohistiocytosis-associated involvement of the central nervous system. J. Infect. Chemother. 24, 292-297 (2018).

30. Deng, B. et al. Cytokine and chemokine levels in patients with severe fever with thrombocytopenia syndrome virus. PLoS ONE 7, e41365 (2012).

31. Liu, J. et al. Dynamic changes of laboratory parameters and peripheral blood lymphocyte subsets in severe fever with thrombocytopenia syndrome patients. Int. J. Infect. Dis. 58, 45-51 (2017). 


\section{Acknowledgements}

This work was supported by the Korea Health Technology R\&D Project, funded by the Ministry of Health \& Welfare, Republic of Korea (HI15C2817), National Research Foundation of Korea (NRF-2017K2A9A1A01092932) to Y.K.C., National Institute of Health (AI129496, AI40718 and AI140705) to J.U.J. and the Ministry of Health \& Welfare, Republic of Korea (HI15C2888) to M.-S.S. We thank I.-P. Mo and K.-S. Kim (College of Veterinary Medicine, Chungbuk National University) for their kind support in the immunohistochemistry assay and the haematoxylin and eosin histopathological study.

\section{Author contributions}

S.-J.P., Y.-I.K. and Y.K.C. conceptualized the study. S.-J.P., Y.-I.K., H.-I.K., E.-H.K., Y.-J.S., C.-H.L., A.P., K.J., W.-J.S., M.-S.S., Y.C. and J.Z. conducted the investigation. S.-J.P., J.U.J. and Y.K.C. wrote the paper.

\section{Competing interests}

The authors declare no competing interests.

\section{Additional information}

Supplementary information is available for this paper at https://doi.org/10.1038/ s41564-018-0317-1.

Reprints and permissions information is available at www.nature.com/reprints. Correspondence and requests for materials should be addressed to J.U.J. or Y.K.C.

Publisher's note: Springer Nature remains neutral with regard to jurisdictional claims in published maps and institutional affiliations.

(C) The Author(s), under exclusive licence to Springer Nature Limited 2018 


\section{Reporting Summary}

Nature Research wishes to improve the reproducibility of the work that we publish. This form provides structure for consistency and transparency in reporting. For further information on Nature Research policies, see Authors \& Referees and the Editorial Policy Checklist.

\section{Statistical parameters}

When statistical analyses are reported, confirm that the following items are present in the relevant location (e.g. figure legend, table legend, main text, or Methods section).

$\mathrm{n} / \mathrm{a} \mid$ Confirmed

$\square$ The exact sample size $(n)$ for each experimental group/condition, given as a discrete number and unit of measurement

$\square$ \ An indication of whether measurements were taken from distinct samples or whether the same sample was measured repeatedly

$\square$ The statistical test(s) used AND whether they are one- or two-sided

Only common tests should be described solely by name; describe more complex techniques in the Methods section.

$\square$ A description of all covariates tested

$\square$ \A description of any assumptions or corrections, such as tests of normality and adjustment for multiple comparisons

$\square$ A full description of the statistics including central tendency (e.g. means) or other basic estimates (e.g. regression coefficient) AND

$\triangle$ variation (e.g. standard deviation) or associated estimates of uncertainty (e.g. confidence intervals)

$\square$ For null hypothesis testing, the test statistic (e.g. $F, t, r$ ) with confidence intervals, effect sizes, degrees of freedom and $P$ value noted

Give P values as exact values whenever suitable.

Х $\square$ For Bayesian analysis, information on the choice of priors and Markov chain Monte Carlo settings

Х $\square$ For hierarchical and complex designs, identification of the appropriate level for tests and full reporting of outcomes

$\square \bigotimes$ Estimates of effect sizes (e.g. Cohen's $d$, Pearson's $r$ ), indicating how they were calculated

$\square$ Clearly defined error bars

State explicitly what error bars represent (e.g. SD, SE, CI)

Our web collection on statistics for biologists may be useful.

\section{Software and code}

Policy information about availability of computer code

Data collection No commercial or open source was collected and used. All data was collected through experimental findings.

Data analysis All statistical analyses were performed using GraphPad Prism version 5.00 or 8.00 for Windows (GraphPad Software, La Jolla, CA). The $n$ values in the figure legend indicate the number of independent experiments and each experiment was conducted with two to three technical replicates. Where appropriate, column analyses were performed using an unpaired, two-tailed t-test. P values less than 0.05 (95\% confidence interval) were considered significant). RNA seq data were analyzed using CLC (QIAGEN) with p<0.05 and QIAGEN's IPA (QIAGEN). The canonical pathways and functional processes Pathway enrichment p-values (Fisher's exact test) and activation z-scores were calculated by IPA.

For manuscripts utilizing custom algorithms or software that are central to the research but not yet described in published literature, software must be made available to editors/reviewers upon request. We strongly encourage code deposition in a community repository (e.g. GitHub). See the Nature Research guidelines for submitting code \& software for further information. 
Policy information about availability of data

All manuscripts must include a data availability statement. This statement should provide the following information, where applicable:

- Accession codes, unique identifiers, or web links for publicly available datasets

- A list of figures that have associated raw data

- A description of any restrictions on data availability

\section{Field-specific reporting}

Please select the best fit for your research. If you are not sure, read the appropriate sections before making your selection.

$\bigotimes$ Life sciences $\quad \square$ Behavioural \& social sciences $\square$ Ecological, evolutionary \& environmental sciences

For a reference copy of the document with all sections, see nature.com/authors/policies/ReportingSummary-flat.pdf

\section{Life sciences study design}

All studies must disclose on these points even when the disclosure is negative.

Sample size No statistical method was used to predetermine sample size. Sample sizes were selected based on previous experience to obtain statistical significance and reproducibility.

Data exclusions No data has been excluded.

Replication All experiments were performed with at least three biological triplicates. All the experimental findings were reliably reproduced.

Randomization Samples were allocated into experimental groups according to their genotype. For all animal studies, mice and ferrets were randomly assigned to each group but by age.

Blinding The investigators were not blinded to group allocation during the collection of specimens from animal, but were binded to data analysis.

\section{Reporting for specific materials, systems and methods}

Materials \& experimental systems

\begin{tabular}{l}
\hline Involved in the study \\
$\square$ Unique biological materials \\
$\square$ Antibodies \\
$\square$ Auman research participants
\end{tabular}

\section{Antibodies}

\begin{tabular}{l|l}
\multicolumn{2}{l}{ Methods } \\
\hline n/a Involved in the study \\
$\triangle$ \\
$\square$ ChIP-seq \\
$\square$ Flow cytometry
\end{tabular}

Antibodies used

Validation
Primary antibody: in-house generated NP monoclonal antibody against SFTSV

Second antibody : HRP-conjugated anti mouse Ig G antibody (cat \#115-035-146, lot \# 126768, Jackson ImmunoResearch). Antibody was used at 1:1000 for IHC.

Primary NP antibody was made in our laboratory and was validated through western blotting and immuno fluorescence assay. Anti-mouse antibody validated by Jackson ImmunoResearch was from Jackson ImmunoResearch. Each antibody was used at 1:1000 dilution for IHC. 
Policy information about cell lines

Cell line source(s)

Vero E6 cells (ATCC No. CRL-1586; American Type Culture Collection, Manassas, VA) were cultured in Dulbecco's Modified Eagle Medium (DMEM; Gibco, Grand Island, NY) containing 2\% fetal bovine serum (FBS; Gibco) with penicillin (100 U/ml) and streptomycin (100 $\mathrm{\mu g} / \mathrm{ml} ; \mathrm{P} / \mathrm{S}$, Gibco) placed in $37^{\circ} \mathrm{C}$ incubator supplemented with $5 \% \mathrm{CO} 2$.

Authentication

Vero E6 cells were purchased from ATCC (No. CRL-1586; American Type Culture Collection, Manassas, VA)

Mycoplasma contamination

All cells were mycoplasma-free.

Commonly misidentified lines

(See ICLAC register)

No commonly misidentified cell lines were used.

\section{Animals and other organisms}

Policy information about studies involving animals; ARRIVE guidelines recommended for reporting animal research

Laboratory animals

Eight-week-old or aged ( $\geq 20$ months) female mice $(n=17)$ of each strain (BALB/c, C3H, C57BL/6, and FVB) were used for SFTSV infection. Young adults (20-24 months, $\leq 2 Y$ ) and aged ferrets (48-50 months), $\geq 4$ Y) were also used for SFTSV infections (14 male and 32 female ferrets were used for this study). All animal experiment protocols were approved by the Medical Research Institute, a member of Laboratory Animal Research Center of Chungbuk National University (LARC) (approval number: CBNUA-986-16-01), and conducted in BSL3 facility (KCDC-14-3-07).

Wild animals

Field-collected samples
No wild animal was used in this study

This study did not involves samples collected from the field. 